\title{
Tangence
}

\section{Réception, narration et tradition dans les écrits autobiographiques africains}

\section{Suzanne Crosta}

Numéro 45, octobre 1994

Authenticité et littérature personnelle

URI : https://id.erudit.org/iderudit/025825ar

DOI : https://doi.org/10.7202/025825ar

Aller au sommaire du numéro

Éditeur(s)

Tangence

ISSN

0226-9554 (imprimé)

1710-0305 (numérique)

Découvrir la revue

Citer cet article

Crosta, S. (1994). Réception, narration et tradition dans les écrits autobiographiques africains. Tangence, (45), 61-73.

https://doi.org/10.7202/025825ar d'utilisation que vous pouvez consulter en ligne.

https://apropos.erudit.org/fr/usagers/politique-dutilisation/ 


\title{
Réception, narration et tradition dans les écrits autobiographiques africains Suzanne Crosta
}

La littérature pour nous ne se répartira pas en genres mais impliquera toutes les approches des sciences humaines.

\author{
Édouard Glissant, \\ Le discours antillais ${ }^{1}$
}

Tout discours sur l'autobiographie, ses conditions, ses particularités, ses types de discours, ses modes d'énonciation suppose que ce genre soit identifiable et même opérant dans les textes qui appartiennent à ce répertoire. Subsumer les écrits de type autobiographique ${ }^{2}$ provenant de divers contextes colonial, néo/postcolonial des littératures francophones de l'Afrique ${ }^{3}$ sous ce genre est une tâche d'autant plus téméraire que les processus externes et internes y influant et dans lesquels se manifestent les spécificités socio-culturelles nous obligent à dépasser les frontières des théories littéraires. Le terme même d'autobiographie pose problème car il faut prendre garde aux trois traits distinctifs qu'elle valorise : le sujet, le récit de vie et l'écriture ${ }^{4}$. Puisque les notions de l'être et les fonctions de l'écriture sont pensées autrement (tout est relatif), il s'ensuit que la prise de parole des écrivains

1 Édouard Glissant, Le discours antillais, Paris, Seuil, 1981, 133.

2 Faute de meilleur terme ou de grille d'analyse, notre concept d'écrit autobiographique se veut malléable et ouvert afin d'inclure ces textes qui rompent les conventions et/ou les contrats de lecture associés à ce genre.

3 Étant donné les limites de cette étude, on s'en tiendra à l'Afrique subsaharienne francophone. Sur le caractère multiculturel et multilingue des sociétés africaines en question, voir l'excellente étude de Kwame Anthony Appiah dans In my Father's House, Oxford/New York, Oxford UP, 1992, en particulier le chapitre intitulé "The Invention of Africa * p. 3-27.

4 L'étude de James Olney, "The Value of Autobiography for Comparative Studies: African vs Western Autobiography", dans African American Autobiography, sous la direction de William L. Andrews, New Jersey, Prentice Hall, 1993 , p. 212-223, s'est penchée précisément sur cette question dans son analyse comparée de Black Boy de Richard Wright et de L'enfant noir de Camara Laye. 
africains ${ }^{5}$ influe sur les traits constitutifs du genre autobiographique tel qu'il se conçoit et se pratique dans les traditions littéraires européennes.

L'autobiographie se penchant sur le récit d'un individu perd ses titres de noblesse dans des contextes où prévaut la communauté et où la fonction de porte-parole à laquelle les écrivains sont appelés à souscrire est de premier ordre. Sur le plan formel, les contrats de lecture figurant dans le péritexte (titre, maison d'édition, dédicace, préface, etc.) ne sont pas toujours concluants ${ }^{6}$. Les signataires ne respectent pas toujours les règles du pacte ${ }^{7}$. On aurait tout lieu de revoir les traits constitutifs de l'autobiographie afin d'accentuer entre autres les lieux interdépendants et intersignificatifs de l'identification personnelle et collective, la recherche d'une référence, la place de l'expressivité et de la parole à l'intérieur des cultures africaines pour y discerner les relations et les correspondances qui sous-tendent les pratiques et les stratégies discursives et narratives des écrivains africains. Le contexte sociologique d'où sont issus les écrits autobiographiques n'est pas non plus non négligeable car la médiation des signes et des normes y transparait tant au niveau de la production qu'au niveau de la signification ${ }^{8}$.

5 Le corpus sur lequel porte ce travail ne prétend aucunement à l'exhaustivité mais se veut un point de départ pour balayer le champs et soulever quelques problèmes quant à une classification générique des écrits autobiographiques africains dont la plupart sont recensés dans le Dictionnaire des œutres littéraires négro-africaines de langue française, Sherbrooke, Naaman, 1983, sous la rubrique "Autobiographie" (p. 660) à savoir: Nafissatou Diallo, De Tilène au Plateau, Dakar, N.E.A., 1975; Lisembe Elebe, Souvenirs d'enfance, Paris, Ia Pensée universelle, 1975; N. G. M. Faye, Le débrouillard, Paris, Gallimard, 1964; Seydou Traoré, 25 ans d'escaliers ou la vie d'un planton, Dakar, N.E.A., 1975; Robert Zotoumbat, Histoire diun enfant trouvé, Yaoundé, CLÉ, 1971. Seront ajoutés Force Bonté de Bakary Diallo (Paris, Rieder, 1926); L'enfant noir de Camara Laye (Paris, Plon, 1953) et Femme d'Afrique d'Aoua Kéita (Paris, Présence africaine, 1975) malgré les ambiguiités de leur statut générique.

6 Voir à ce sujet, l'étude d'Hélène Jaccomard et de Jean-Marie Volet, "Pacte autobiographique et écrivaines francophones d'Afrique noire, "Prêsence francophone, 41, 1992, p. 9-26.

7 La notion du "pacte" ou du "contrat" proposée par Philippe Lejeune dans Moi aussi, Paris, Seuil, 1986, p. 13-35, est problêmatique non seulement en raison des manceuvres des trois signataires (éditeur, écrivain et lecteur) mais aussi en raison des enjeux discursifs et narratifs qui influent sur la conceptualisation et l'articulation du " je ".

8 Cf. Martin Kohli, "Biography: Account, Text, Method," dans Biograpby and Society: The Life History Approach in the Social Sciences, sous la direction de D. Bertaux, Londres, Sage Publications, 1981, p. 61-75. 
Le faible accueil réservé aux textes de notre corpus, à quelques exceptions près, accuse les conditions de production et de réception où la médiation des institutions et des normes de lectures jouent sur les paliers significatifs des écrits autobiographiques. Il est pertinent de relever que notre corpus est composé de textes écrits en français et de ce fait même découle leur marginalisation ou leur élitisme 9. L'analphabétisation et l'hétérogénéité culturelle et linguistique qui caractérisent la situation de maints pays africains influent sur le statut mineur des écrits autobiographiques d'autant plus que les impératifs politiques de la littérature entrent en jeu. Destinés pour l'exportation en raison du choix de langue, ces textes n'echappent pas aux configurations socio-politiques et institutionnelles du contexte de réception. Paradoxalement, le public étranger auquel s'adresse ces ouvres ne partage pas toujours la sensibilité des écrivains; distance qui se manifeste aussi chez les éditeurs ${ }^{10}$. L'excentrement ${ }^{11}$ du sujet motive très souvent une explication dans le péritexte. À titre d'exemple, la description du récit se trouvant au dos de la page de couverture oriente l'axe du Débrouillard de Faye non vers l'intention de l'auteur sénégalais mais vers les attentes du lecteur français. La dévalorisation du pays d'origine de l'auteur et la glorification du pays d'accueil ne laissent aucun doute sur l'égalité des relations entre l'éditeur, l'auteur et le lecteur. Ici, la signature de l'éditeur sanctionne une lecture axiologique qui déforme la signification du récit pour neutraliser ou normaliser son impact socioculturel et politique. Il ne s'agit plus d'un récit de vie d'un champion de boxe mais le récit d'un homme régénéré par son contact avec la France. La circulation textuelle et la motivation

9 Cf. Frantz Fanon, Les damnés de la terre, Paris, Maspero, 1968, p. 141-175; Ngugi wa Thiong'o, Decolonising the Mind, London, J. Currey, 1986, p. 2027.

10 L'aveu de Louis Gardel, conseiller éditorial au Seuil, est révélateur à cet égard: "En raison de mes propres centres d'intérêt, je reçois énormément ce que j'appelle des "souvenirs pieux" de Maghrébins de toutes origines, pieds-noirs, arabes, juifs. J'ai l'impression qu'il n'y a pas un pied-noir qui écrive sans m'envoyer son manuscrit! Je trouve ça normal, mais le fait même d'en recevoir beaucoup me rend très sévère. Dans un autre style, ce qui m'encombre aussi, ce sont les manuscrits autobiographiques, très douloureux et sans recul, parfois même accompagnés de menaces de suicide au téléphone. Il faut autre chose qu'une grande douleur pour faire un écrivain." Lire, avril 1994, p. 29.

11 J'emprunte ce néologisme à Maximilien Laroche. Voir son étude intitulée "Excentrement-décentrement, recentrement-autocentrement, * Francographies, $\mathrm{n}^{\circ}$ spécial II, 1993, p. 17-27. 
64

idéologique sont des facteurs à retenir pour saisir l'intérêt des écrits autobiographiques dans le contexte de réception.

Bref, les enjeux idéologiques sous-jacents à la production et à la réception des textes autobiographiques limitent l'accessibilité à l'institution ou plutôt recentrent l'axe de la lecture avant de garantir l'adhésion à l'institution littéraire. Cette détermination et cette édification institutionnelle de la "subjectivité" au sens de Roland Barthes ont poussé certains spécialistes et critiques littéraires provenant des contextes néo/post-coloniaux à nager à contre-courant. Said nous suggère que la lecture des textes néo/ post-coloniaux se doit être "en contrepoint" afin de dévoiler les contradictions, les paradoxes circonscrivant la signification de ces textes. Il la définit de la manière suivante:

[...] contrapuntal reading must take into account both processes, that of imperialism and that of resistance to it, which can be done by extending our reading of the texts to include what was once forcibly excluded. ${ }^{12}$

Il conviendrait d'ajouter à cette réflexion que cette lecture "en contrepoint. s'avère une démarche de conscientisation, de récupération et de réappropriation des signes et des faits culturels. Cette démarche critique correspond à l'éthique et à la pratique d'écriture de Diallo, de Laye, d'Elebe etc. dont le projet n'est pas de soumettre leurs textes aux pulsions de mort (au sens de Jean Baudrillard) et de réduire le texte à un cimetière où gisent les bien-aimés et les défunts des siens mais de le transformer en chants, en proverbes, en paroles qui assurent la survie personnelle et collective des morts-vivants (au sens de John Mbiti).

Une prise en considération des "sujets" ${ }^{13}$ des récits de vie du corpus nous permet de constater que les écrivains se refusent à

12 Edward Said, Culture and Imperialism, New York, A. Knopf, 1994, p. 66. "[...] une lecture en contrepoint doit tenir compte des deux processus, celui de l'impérialisme et celui de la résistance qui l'accompagne, ce qui peut se faire en élargissant notre lecture des textes jusqu'à inclure ce qui en avait été exclu sous contrainte." [je traduis]

13 Voir les positions divergentes des critiques suivants: Mohamadou Kane, Roman africain et tradition, Dakar, Nouvelles Éditions africaines, 1982; J. P. Makouta M'Boukou, Introduction à l'étude du roman négro-africain de langue française, Dakar, Nouvelles Éditions africaines, 1980; Bernard Mouralis, "African Literature and the Writing Subject, "Research in African Literatures, vol. XXI, $\mathrm{n}^{\circ} 1,1990$, p. 69-78; Léopold Sédar Senghor, Négritude et bumanisme, Paris, Seuil, 1964. 
sortir de l'ordinaire: ce n'est pas de la vie de personnages célèbres qu'il s'agit mais plutôt de la vie quotidienne d'un enfant, d'une sage-femme, d'un boxeur, d'un militaire, d'un planton, etc. La modestie et l'humilité sont exhortées comme en témoigne l'avant-propos De Tilène au Plateau:

Je ne suis pas une héroïne de roman mais une femme toute simple de ce pays: une mère de famille et une professionnelle (sage-femme et puériculturiste [sicl]) à qui sa maison et son métier laissent peu de loisir. (p. 9)

Chaque écrivain(e) se soucie de nous transmettre à partir d'une perspective interne les traditions et les valeurs qui régissent sa communauté. Qu'il s'agisse de Force Bonté ou de Souvenirs d'enfance, tous les textes autobiographiques se démarquent difficilement de leur ancrage temporel et spatial de la production en raison des référents et des valeurs implicites et/ou explicites auxquels ils renvoient et répondent. Cette constante (la présence et l'inscription du collectif) a également des implications épistémologiques. Le discours autobiographique des écrivains à l'étude établit un réseau de connexions avec les discours du savoir dans les sciences humaines de par la mise en représentation d'un sujet qui se cherche et se définit à partir d'un concours de faits contextuels ou pragmatiques qui sont refigurés et réactualisés dans le texte ${ }^{14}$. Les lieux d'expression entre le sujet individuel et le sujet collectif transparaissent dans les écrits autobiographiques où la lecture est ponctuée de mises en garde et de cautions sur les notions du réel (la famille étendue, les valeurs et les normes de leur communauté, les rites et les rythmes de leur existence, les rapports avec les ancêtres et les diverses générations etc.) en fonction de l'acception culturelle du narrateur et/ou de l'auteur en question. Le souci d'objectiver les données de la culture serait à réinterpréter car elles définissent les lieux subjectifs de l'être dans la communauté ${ }^{15}$. Le

14 Voir l'étude de John Mbiti où il souligne l'interrelation significative entre l'individu et la communauté: "Dans la société traditionnelle, l'individu n'existe pas et ne peut pas exister seul, la vie n'est possible que de façon corporative. Chacun est redevable de son existence à d'autres hommes, y compris ceux des générations précédentes, et à ses contemporains. Il n'est qu'une partie du tout. C'est donc la communauté qui a la tâche de faire, de créer ou de produire l'individu, car cet individu dépend du groupe." Religions et pbilosophie africaines, trad, par C. LeFort, Yaoundé, CLÉ, 1972, p. 118-119.

15 Pour exprimer ce rapport entre l'être et la communauté au niveau de la typologie narrative, Olney postule deux néologismes: "autoautography" et "auto 
66

dialogue s'établissant entre le discours autobiographique et les discours scientifiques des divers champs de savoir aurait donc pour but d'élargir les fonctions du texte et de le signifier à travers les signes culturels et artistiques multiples que se donne le projet d'écriture.

Comme nous l'avons constaté, les écrivains de notre corpus invoquent deux impératifs: celui de dire le vrai et celui de dire le "nous", c'est-à-dire tracer les relations entre l'être et la communauté. Le critère de véracité est manifeste aussi bien dans le péritexte que dans le récit. Dans l'introduction du Débrouillard, l'auteur déclare: "Mais croyez-moi, ce n'est pas une histoire gratuite que je vais vous raconter, c'est une réalité, du fait que je l'ai vécue moi-même" (p. 7). Cette préoccupation de relater fidèlement le vécu se transpose également dans les récits de Diallo, de Laye et de Traoré. Quant au critère de solidarité avec la communauté, il se traduit plus souvent sur le plan de la modalité narrative dans la mesure où le "je" s'inscrit dans une relation intersignificative avec le "nous". Cette position est renforcée chez Diallo et Laye par le recours usuel des prolepses (p. 87, 119, 121) pour induire à la réflexion. Bien que les écrivains à l'étude se donnent pour tâche première de nous communiquer les régies et les valeurs de la vie communautaire, leurs prises de position sont divergentes. Force Bonté, L'enfant noir, 25 ans d'escaliers ou la vie d'un planton et Souvenirs d'enfance tracent un univers communautaire idyllique mais en voie de transition. De Tilène au plateau et Femme d'Afrique nous renseignent sur les espaces clos et ouverts de leur communauté. Le débrouillard, par contre, s'en prend aux structures oppressives de sa communauté qui s'est montrée impuissante à pallier les abus de pouvoir de son père. Il est tout de même important de souligner l'attachement du narrateur envers sa mère qui, dans la symbolique du texte, est associée au pays.

Inversement, les écrits autobiographiques à l'étude remettent en question la valeur de l'individualisme d'autant plus que celuici résulte d'une scolarisation où le système de valeurs inculqué à l'enfant est incompatible avec sa formation familiale et sociale. Cette problématique se trouve savamment articulée dans le texte de Diallo, De Tilène au Plateau sous la rhétorique de la gourman-

phylography". Celui-ci décrit les récits où le sujet est en rapport avec la communauté (je = nous) alors que celui-là désigne les narrations où le sujet se situe dans l'histoire de l'écriture du Moi (je = Je). Olney, p. 218. 
dise ${ }^{16}$. S'établit, au fur et à mesure que se déroule le récit, une relation entre la gourmandise et la disparition de cet esprit communautaire que l'on regrette au moment de l'écriture. Cette sensibilité est d'autant plus évidente dans la technique dominante où la narratrice raconte un épisode au passé puis l'actualise en montrant la leçon qu'elle en a tirée ${ }^{17}$. La rhétorique de la gourmandise est inextricablement liée au statut d'orpheline attribué à Safi et à la problématique de l'assimilation culturelle. Le récit se construit sur deux paliers narratifs où la rhétorique de la gourmandise qui caractérise l'existence de la jeune Safi est contrecarrée par une rhétorique de la sagesse de la narratrice adulte. Prédisposée à la gourmandise depuis son enfance, Safí ne parvient pas à assouvir un appétit grandissant qui génère d'autres vices. Ses envies pour les bonbons et les fruits la conduisent au vol et au mensonge tandis que sa prédilection pour les biens matériels provenant de la France (les vêtements, les livres...) entraîne son assimilation culturelle. Il s'ensuit que son père, gardien féroce de la tradition, s'affaiblira et perdra sa voix et sa vie. C'est par le biais de l'écriture que la dévoreuse se transforme en sage-femme. L'exil intérieur projeté par la représentation et la symbolisation de Safi est médiatisé et filtré par le discours de la narratrice dans le but de s'autocentrer et de s'enraciner dans sa communauté.

Les rapports de l'être à la communauté étant multiples en fonction des expériences du narrateur/personnage dans le récit, les rapports entre le sujet et l'écriture sont tout aussi complexes de par les tensions suscitées et de par la fluidité avec laquelle ils construisent leur récit de vie. Dans Le débrouillard, l'auteur préface son texte en s'excusant:

Ce livre n'est pas écrit par un écrivain ni par un professeur. Seulement par une personne de niveau très primaire. Donc je

16 Le projet d'écriture de Diallo concourt avec celui de Birago Diop dans Les nouveaux contes d'Amadou Koumba ou avec celui de Ferdinand Oyono dans Une vie de boy. Il existe certainement un dialogue entre les trois textes surtout pour ce qui est de leur perception et de leur interprétation de la gourmandise. La dimension morale est manifeste chez ces écrivains dans la mesure où la gourmandise est un vice qui singularise alors que le partage est une vertu qui solidarise.

$17 C f$. les lieux et la signification des truchements de la narratrice aux pages 14 , $17,20,37,44,67,93,97,102,104,112,119,125,130,132$. Ceux-ci s'articulent tantôt au présent tantôt au futur et ont pour fonction de montrer la pertinence de ces incidents passés. 
68

m'excuse auprès de vous des fautes de français et de composition. (p. 7)

Écrivain par vocation et non par profession, son rapport à la. langue française et à l'écriture sont tendus et apologétiques. Il en est de même pour Diallo, Elebe, Traoré chez qui cette tension linguistique ne figure pas seulement dans le péritexte mais déborde dans leur récit. Par conséquent, les textes à l'étude révèlent, selon diverses méthodes et formules, les présupposés et le discours normatif du genre autobiographique soit sur le plan de la représentation, sur le plan de la narration ou sur le plan de la symbolisation textuelle.

La référentialité étant plus au moins une constante dans les écrits autobiographiques, il est intéressant de constater les multiples attributions sous lesquelles peut se caser un seul et même texte. Les positions sont parfois extrêmes et tranchantes. Alors que le critique Makouta M'Boukou affirme sans ambages que "L'autobiographie est un genre qui fait fureur à notre époque, dans d'autres contextes culturels, mais pas en Afrique noire" 18 . Renée Larrier soutiendra le contraire, comme en témoigne son étude sur les implications sociales et politiques des récits autobiographiques.

Autobiographical narratives have been a major genre in African francophone literature since the publication of Bakary Diallo's Force Bonté in 1926. ${ }^{19}$

L'étanchéité des positions soulève les contradictions internes et externes quant à la conception et à la praxis sociale de l'autobiographie dans le contexte des littératures africaines. Les champs d'expériences et de connaissances où se situent les textes sont polarisés par des relations d'opposition entre l'individualisme et la solidarité communautaire, entre l'expression esthétique et la fonction utilitaire de la littérature. Si maints critiques des littératures africaines, à l'instar de Mohamadou Kane, Ambroise Kom, J. P. Makouta M'Boukou, Guy Ossito Midiohouan ne s'accordent toujours pas sur le classement des œuvres littéraires et si les sour-

18 J. P. Makouta M'Boukou, Introduction à l'étude du roman négro-africain de langue française, Dakar, N.E.A., 1980, p. 184.

19 L'étude de Renée Larrier, "Autobiographical Authority and the Politics of Narrative ", $S T C L$, vol. $\mathrm{XV}, \mathrm{n}^{\circ} 1,1991, \mathrm{p} .77$. "Les récits autobiographiques sont un genre majeur dans la littérature africaine francophone depuis la publication de Force Bonté de Bakary Diallo en 1926." 
ces bibliographiques se butent à la classification de ces écrits, c'est que le genre autobiographique est non seulement pensé autrement mais diversifié intérieurement. À titre d'exemple, Femme d'Afrique, se trouve classé comme un manifeste politique, un essai, une autobiographie, un mémoire historique; $L e$ débrouillard est à la fois une autobiographie, un document sociologique (sur la boxe), un roman; Force Bonté, L'enfant noir, De Tilène au Plateau, Histoire d'un enfant trouvé, et Souvenirs d'enfance se trouvent casés sous la rubrique autobiographie, roman, roman autobiographique, etc. Or les écrivains de notre corpus pensent le genre en fonction de leur vécu. S'ils ne se conforment pas aux conventions de l'autobiographie telle qu'on la pratique ailleurs, c'est qu'elles ne conviennent pas à la perception ou à l'articulation du réel des écrivains à l'étude. Il faudrait même repenser les termes de "subversion" ou de "transgression" car la non-conformité aux conventions du genre repose sur une volonté de respecter les spécificités culturelles du je-narrant. Il est indéniable que la référentialité telle qu'elle se trouve configurée, refigurée et réactualisée dans les textes à l'étude sert d'indice à la présence des discours sociaux dans l'imaginaire, mais elle n'explique pas de façon satisfaisante les pratiques et les stratégies d'écriture.

Il conviendrait de revoir les spécificités de leur écriture en fonction de la présence et de la pertinence de l'oralité dans la conception et la construction du sujet, des thèmes, des discours se faufilant dans le projet autobiographique des écrivains africains $^{20}$. Une prise en considération des fonctions de l'oralité dans la pratique autobiographique mérite de sérieuses considérations pour relever les modes d'expression de la subjectivité des écrivains africains. Ceci suppose bien sûr qu'il y ait continuité sinon corrélation entre les discours des récits oraux provenant des civilisations orales africaines et ceux des récits écrits provenant des cultures de l'imprimé. Comme nous l'avons relevé, le sujet des écrits autobiographiques à l'étude se trouvant excentré de l'histoire littéraire et des conventions qui lui sont a priori prescrites par les "lois" du genre, il s'acharne à négocier un espace où

20 Il faut à présent problématiser le point de départ de cette analyse en raison des limites de son postulat. Se concentrer sur les textes autobiographiques, c'est privilégier l'écriture et exclure d'autres modes d'expressions artistiques et langagières qui pourraient remplir cette même fonction. 
peuvent se mouvoir, s'articuler et s'accommoder plusieurs traditions provenant de différentes aires culturelles. Maints critiques littéraires travaillant dans le champ de l'oralité dans divers contextes culturels de l'Afrique ont montré les différents degrés d'investissement auxquels s'engagent les écrivains pour faire signifier les particularités et les formes relevant de la narration orale 21 .

Le texte de Robert Zotoumbat nous fournit à cet égard un cas intéressant à examiner. Le texte se présente et se lit comme étant "en partie autobiographique". La fluidité générique délibérée influe sur le contrat de lecture que nous proposent l'éditeur et le narrateur dans la mesure où le lecteur, pour saisir les effets sémantiques du texte, doit pouvoir se déplacer au gré des référents évoqués. Dans la section "Présentation", le narrateur avoue qu'il lui est difficile de parler de sa vie et sollicite un vieil ami, nommé Ngoye, de partager son récit de vie. Le narrateur cède sa voix à Ngoye dont la prise de parole glisse tout naturellement au niveau de la narration. Mais on apprend aussi que le récit de Ngoye trouve son support dans le récit d'une vieille femme. Ngoye avoue au narrateur/auditeur que "Ce fut cette vieille qui me raconta tous ces faits que je viens de te livrer." (p. 49) La mise en représentation de la situation narrative orale établit tout un réseau de connivences qui démantèle l'autorité narrative et transforme la fonction du narrateur en griot, c'est-à-dire, en un maitre de parole(s). C'est à lui qu'incombe la tâche de donner un statut aux voix et aux souffles (la disposition structurelle) de son récit. Ajoutons que la prise de parole du narrateur se solidarise avec celle d'une lignée de conteurs qui l'aideront à articuler ou plutôt à symboliser le "malêtre" qu'il ressent à raconter sa vie: "Ma vie! Vraiment je risque de vomir tripes et boyaux en prononçant ce mot, tant il me rappelle de choses répugnantes." (p. 10) La déclaration du narrateur suggère une polarisation entre le jescripteur et le je-narrant où l'arbitraire du langage empiète sur le

21 Nous soulignons entre autres les travaux pionniers des chercheurs suivants: Bassirou Dieng, "Narrative Genres and Intertextual Phenomena in the Sahelian Region," Research in African Literatures, vol. XXIV, n ${ }^{\circ} 2,1993$, p. 33-45; Ruth Finnegan, Oral Literature in Africa, Oxford et Nairobi, Oxford UP, 1971 ; Mohamadou Kane, Roman africain et tradition, Dakar, N.É.A., 1982; Amadou Koné, "Roman africain et littérature orale," Komparatistiscbe Hefte $n^{\text {os }} 15-16,1987$, p. 27-36; Emmanuel Obiechina, Culture, Tradition and Society in the West African Novel, Cambridge, Cambridge UP, 1975; Isodore Okpewho, The Epic in Africa, New York, Columbia UP, 1979. 
désir de penser et de rêver l'autre en lui et hors-de-lui vu les leurres et les vicissitudes de son existence. Le récit se pose comme gageure de cette quête de vie: "Lui parlerai-je à mon tour de la mienne?" (p. 10) Le recours à un conteur (Ngoye) vise à faciliter la parole en établissant un double qui projetterait un récit de vie qui l'engagerait à lui révéler la sienne.

Le démantèlement de l'autorité narrative et l'absence d'une identification explicite (l'emploi du nom propre de l'auteur) n'ont pas pour seul but de baliser les conventions et les critères formels de l'autobiographie mais de soulever la problématique de l'objectivité historique qui normalise l'oppression et la souffrance à des faits. Le récit personnel de Ngoye est, en effet, une recherche des origines, du sens de l'existence humaine lorsque les lieux de l'identification personnelle sont enfouis. Pour s'autocentrer, narrateur, conteur, personnage fusionnent dans la parole de sorte que les pôles sujet/objet sont inopérants dans la recherche identitaire du narrateur. Le je-narrant étant incapable de relater sa vie a besoin d'un autre "je" pour démarrer le récit. Ce saut entraîne des déplacements multiples du lecteur pour identifier et signifier les référents. S'instaure donc un effet d'étrangeté qui s'articule dans l'excentrement du narrateur et l'autocentrement du personnage Ngoye qui apprend son surnom: "Moana-wa-za" [l'enfant de la famine] (p. 49).

Le jeu des distances entre sujets et récits de vie témoigne de ce va-et-vient entre deux espaces qui ne sont pas exclusifs, mais intersignificatifs, à la formation identitaire du sujet. Le lecteur est censé suivre l'exemple du narrateur qui se met à l'écoute de Ngoye, vieux conteur, détenteur du mémoriel, chargé d'historiciser sa vie personnelle et à la fois collective rapportée à la première personne. La complicité entre le narrateur et le conteur Ngoye a pour effet d'inscrire la quête de sens dans la situation de la narration orale mise en scène. L'écriture se plierait donc aux exigences de la parole du conteur dont le récit de vie répartit le texte écrit en y donnant la disposition et la structure actuelle.

Par ailleurs, le recours à une chaîne de conteurs problématise le contexte de réception de l'œuvre dans laquelle le destinataire est construit dans le texte ${ }^{22}$. Dans Histoire d'un enfant trouvé, le

22 La critique littéraire a beaucoup cogité sur le penchant autobiographique d'Une si longue lettre de Mariama Bâ mais la classification de ce texte pose 
72

récit de la vieille est destiné à Ngoye, tout comme le récit de celui-ci est adressé au narrateur qui réserve son récit à ceux qui veulent bien tendre leurs oreilles à cette chaîne sonore. Cet accent sur l'écoute est également évoqué dans l'introduction du Débrouillard où l'auteur ne se réfère pas à ses lecteurs mais à ses "auditeurs et auditrices". La représentation de la situation dialogique ou de la situation communicationnelle est aussi inscrite dans les textes d'Elebe, de Kéita et de Traoré. Dans Souvenirs d'enfance d'Elebe, le narrateur rapporte de très longs témoignages attribués pour la plupart à sa mère et aux membres de sa famille étendue. La gageure de maintenir l'illusion de vérité au niveau de la narration cède à la volonté du narrateur de céder sa voix aux siens. C'est par le biais de leurs témoignages que le récit de vie du narrateur se construit et acquiert son sens car si parler de sa communauté c'est parler de soi, il devient tout aussi possible de constater que quand la communauté parle d'elle-même, elle parle de lui. En effet, dans Souvenirs d'enfance le narrateur au tout début du récit cède sa voix à sa mère qui parlera des circonstances de la naissance, de la scolarisation et de la vie quotidienne du narrateur. Ainsi les siens sont-ils garants de son récit de vie, car ils servent à compléter les lacunes de sa mémoire individuelle. Le témoignage des siens accuse le caractère subjectif de tout récit de vie individuelle de par les trous de mémoire qui ont besoin d'être comblés.

Au terme de cette étude, il conviendrait de reposer les jalons qui ont marqué les étapes de notre réflexion. D’abord les critères de généricité sont délibérément rendus inopérants sinon flous et renversent les structures qui régissent le sujet, le récit et l'écriture autobiographiques. L'auteur se trouve dans la situation paradoxale de se frayer un espace textuel où peut figurer la communauté dans un genre qui, dans son usage courant, est le lieu exclusif d'un sujet individuel. En conséquence, il s'opère dans les écrits autobiographiques, à des degrés divers, un désir d'établir une continuité à partir des fragments "éperdus" et/ou épars de la

problème tout comme celui de Zotoumbat. Le recours à une lectrice interne explique en partie les modifications que l'auteure a effectuées ici à la forme épistolaire. La destinataire - le lectorat sénégalais féminin, symbolisé par Aissatou - est appelée à se solidariser avec la narratrice pour expliquer au lectorat français/francophone les traditions et les coutumes sénégalaises qui ont contribué au maintien de l'infériorisation de la femme. 
vie: d'où le recours à l'oralité, la recherche d'une référence autre; d'où la valorisation d'une prise de parole qui se veut solidaire de sa communauté et d'une nouvelle pratique d'écriture puisant dans les traditions esthétiques de leur société. L'excentrement du sujet se trouve ainsi renversé par une volonté de recentrer la trajectoire de sa recherche et de son écriture autour de l'inscription moyennant les particularités et les discours relevant des traditions, des mythes fondateurs, des proverbes qui, dans le texte, permettent au sujet de reconnaittre les lieux de son identification à travers une saisie de l'univers de son être. L'interdiscursivité entre les champs des sciences humaines, manifeste dans la transposition et les transferts des discours provenant de différentes traditions culturelles, sert à contrecarrer le statut d'orphelinat dans lequel sont relégués les écrits coloniaux, néo/post-coloniaux de l'Afrique. Ainsi l'imaginaire et les lieux de l'identification du "je" puisent-ils dans le réel mais sans en être prisonniers, car l'écriture, travaillée par les signes culturels et littéraires de l'oralité, oscille entre autobiographie et conte, autobiographie et épopée (entre autres le "mvett" 23 ) entre autobiographie et légende, et se transforme en une poétique d'expressions et/ou de connexions toujours à rechercher, à dynamiser ou à réactualiser.

23 Voir entre autres les travaux de Tsira Ndong Ndoutoume, Le Mvett, épopée fang, Paris, Présence africaine, 1984, et de Pascal Boyer, Barricades mystérieuses et pièges à pensée. Introduction à l'analyse des énoncés fang, Paris, Société d'ethnologie, 1988. 